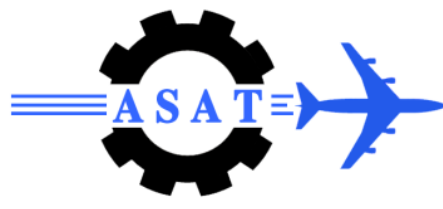

\title{
Fast Converging with High Accuracy Estimates of Satellite Attitude and Orbit Based on Magnetometer Augmented with Gyro, Star Sensor and GPS via Extended Kalman Filter
}

\author{
T. Habib
}

\begin{abstract}
The primary goal of this work is to extend the work done in, [1], to provide high accuracy satellite attitude and orbit estimates needed for imaging purposes and also before execution of spacecraft orbital maneuvers for the next Egyptian scientific satellite. The problem of coarse satellite attitude and orbit estimation based on magnetometer measurements has been treated in the literature. The current research expands the field of application from coarse and slow converging estimates to accurate and fast converging attitude and orbit estimates within $0.1^{\circ}$, and $10 \mathrm{~m}$ for attitude angles and spacecraft location respectively $(1-\sigma)$. The magnetometer is used for both spacecraft attitude and orbit estimation, aided with gyro to provide angular velocity measurements, star sensor to provide attitude quaternion, and GPS receiver to provide spacecraft location. The spacecraft under consideration is subject to solar radiation pressure forces and moments, aerodynamic forces and moments, earth's oblateness till the fourth order (i.e. $J_{4}$ ), gravity gradient moments, and residual magnetic dipole moments. The estimation algorithm developed is powerful enough to converge quickly (actually within 10 seconds) despite very large initial estimation errors with sufficiently high accuracy estimates.
\end{abstract}

Keywords: : Fast Convergence, Attitude, Orbit, High Accuracy, Estimation, Magnetometer, Gyro, Star Sensor GPS, Earth Oblateness, Drag, Solar Radiation, Residual Dipole, Extended Kalman Filter.

\section{Introduction}

Magnetometer has the advantages of low cost, high reliability, non-intermittent measurements (compared to sun-senor which couldn't be used during eclipse times), and long lifetime due to the absence of moving parts inside. On the other hand, magnetometer measurements couldn't be used for instantaneous spacecraft attitude determination process. This is because attitude determination algorithms found in Refs. [2], [3] (such as q-method, algebraic method, optimized triad, modified algebraic method, etc.) require at least two or more sensors that measure more than a single physical quantity. In order to use measurements of a single physical quantity (such as the earth's magnetic field measured by a three-axis magnetometer) estimation algorithms (which are by default model based) must be used instead. The problem of coarse satellite attitude and orbit estimation based on magnetometer measurements has been treated in the literature [1]. In this treatment, necessary derivations to reveal the algorithms found in [4], and [5] have been made.

\footnotetext{
Researcher, Dynamics and Control Department, National Authority for Remote Sensing and Space Sciences, tamermekky@ hotmail.com
} 
The magnetometer is used mainly to provide measurements during the detumbling and the standby mode of operation which by definition don't require high accuracy attitude or orbit estimates. The nature of the estimation process of satellite attitude and orbit based on magnetometer is characterized by slow convergence (typically requires several revolutions about the earth) and low accuracy estimates (of about $5^{\circ}$ to $7^{\circ}$ ). During high accuracy satellite operation mode, magnetometer is used mostly for attitude estimation [6], [7]. Inclusion of magnetometer measurements for spacecraft orbit estimation in addition to attitude estimation during high accuracy operation mode is considered to be a challenge. The resulting solution enhances attitude and orbit estimation errors that are incorporated with standard estimation techniques. The primary goal of this work is to provide fast converging and high accuracy satellite attitude and orbit estimates needed for imaging purposes and also before execution of spacecraft orbital maneuvers for the next Egyptian scientific satellite despite large initial estimation errors. The current research expands the field of attitude and orbit estimation based on magnetometer from coarse and slow converging to accurate (typically within $0.1^{\circ}$, and $10 \mathrm{~m}$ for attitude angles and spacecraft location respectively $1-\sigma)$ and fast converging (actually within 10 seconds) attitude and orbit estimates. The magnetometer is used for both spacecraft attitude and orbit estimation, aided with gyro to provide angular velocity measurements, star sensor to provide attitude quaternion, and GPS to provide spacecraft location. The spacecraft under consideration is subject to many disturbances such as solar radiation pressure forces and moments, aerodynamic forces and moments, earth's oblateness till the fourth order (i.e. $J_{4}$ ), gravity gradient moments, and residual magnetic dipole moments. Large initial attitude estimation errors typically about $\left(180^{\circ}\right.$ for the yaw angle, $175^{\circ}$ for the roll and $85^{\circ}$ for the pitch angle) are used to test the convergence of the estimation algorithms from nearly lost in space conditions. The estimation algorithm developed is powerful enough to converge quickly (actually within 10 seconds) despite very large initial estimation errors with sufficiently high accuracy predictions.

\section{Modeling Spacecraft Dynamics}

The nonlinear differential equations describing the combined translational and rotational motions of the satellite are given by [1],

$$
\stackrel{o}{X}=\left[\begin{array}{l}
o \\
X_{O} \\
o \\
X_{A}
\end{array}\right]=\left[\begin{array}{ll}
f_{11} & f_{12} \\
f_{21} & f_{22}
\end{array}\right]\left[\begin{array}{l}
X_{O} \\
X_{A}
\end{array}\right]+\left[\begin{array}{l}
B_{O} \\
B_{A}
\end{array}\right]=f(X)+B
$$

with

$$
\begin{aligned}
& f_{11}=\left[\begin{array}{cc}
0_{3 \times 3} & I_{3 \times 3} \\
\frac{-\mu_{E}}{\left\|R_{I}\right\|^{3}} I_{3 \times 3} & 0_{3 \times 3}
\end{array}\right], \quad f_{12}=0_{6 \times 7}, \quad f_{21}=0_{7 \times 6}, \quad f_{22}=\left[\begin{array}{cc}
\frac{1}{2} \Omega & 0_{4 \times 3} \\
0_{3 \times 4} & J^{-1}\left[\left(J \omega+H_{w}\right) \times\right]
\end{array}\right], \quad B_{O}=\left[\begin{array}{c}
0_{3 \times 1} \\
a_{I}
\end{array}\right], \quad \text { and } \\
& B_{A}=\left[\begin{array}{c}
0_{4 \times 1} \\
J^{-1} M
\end{array}\right]
\end{aligned}
$$

where

$$
\begin{array}{ll}
0_{i \times j} & : \text { is an } i \times j \text { zero matrix. } \\
I_{i \times j} & : \text { is an } i \times j \text { unit matrix. } \\
\mu_{E} & : \text { is the earth's gravitational constant }\left(\mu_{E}=3.986 \times 10^{14} \mathrm{~m}^{3} / \mathrm{s}^{2}\right) . \\
\Omega & : \text { is the skew symmetric matrix of the inertial angular velocities defined by }
\end{array}
$$


$\Omega=\left[\begin{array}{cccc}0 & \omega_{z} & -\omega_{y} & \omega_{x} \\ -\omega_{z} & 0 & \omega_{x} & \omega_{y} \\ \omega_{y} & -\omega_{x} & 0 & \omega_{z} \\ -\omega_{x} & -\omega_{y} & -\omega_{z} & 0\end{array}\right]$

$J \quad$ : is the spacecraft inertia matrix given by

$J=\left[\begin{array}{ccc}J_{x x} & -J_{x y} & -J_{x z} \\ -J_{y x} & J_{y y} & -J_{y z} \\ -J_{z x} & -J_{z y} & J_{z z}\end{array}\right]$.

$H_{w} \quad$ : is the angular momentum of the wheels (the case study at hand has a momentum wheel mounted in the pitch direction).

$[\gamma \times]:$ is the cross product matrix of $[\gamma]=\left[\begin{array}{lll}\gamma_{x} & \gamma_{y} & \gamma_{z}\end{array}\right]^{T}$ calculated from

$[\gamma \times]=\left[\begin{array}{ccc}0 & -\gamma_{z} & \gamma_{y} \\ \gamma_{z} & 0 & -\gamma_{x} \\ -\gamma_{y} & \gamma_{x} & 0\end{array}\right]$

$a_{I} \quad:$ is the input inertial acceleration.

$M \quad:$ is the input torque.

The state vector, $X$, of the spacecraft dynamics is chosen as $X=\left[\begin{array}{ll}X_{O}^{T} & X_{A}^{T}\end{array}\right]^{T}$ with $X_{O}=\left[\begin{array}{llllcc}X_{I} & Y_{I} & Z_{I} & X_{I} & Y_{I} & Z_{I}\end{array}\right]^{T}$ defined as the orbital state vector comprised from inertial position and velocity components respectively, and $X_{A}=\left[\begin{array}{lllllll}q_{1} & q_{2} & q_{3} & q_{4} & \omega_{x} & \omega_{y} & \omega_{z}\end{array}\right]^{T}$ is the attitude state vector composed from the quaternion vector with $q_{4}$ representing the real component of the attitude quaternion (representing the rotation from inertial to body axes), and the inertial angular velocities. The case study satellite is subject to many disturbances such as solar radiation pressure forces and moments, aerodynamic forces and moments, earth's oblateness till the fourth order (i.e. $J_{4}$ ), gravity gradient moments, and residual magnetic dipole moments. All these models are propagated through equation (1) for the true and estimated spacecraft but not included in the propagation of the state transition matrix calculated by the extended Kalman filter algorithm. This is because of several factors:

1- The motion of the spacecraft is mostly modeled by equation (1).

2- The disturbances presented are too high nonlinear functions of the states which impose many complications over (and even could inhibit) the computation of the derivatives needed by the state transition matrix with approximately no gain for the accuracy.

3- The complete effect of disturbance forces and moments is taken into consideration during state propagation of the extended Kalman filter.

4- The state propagation matrix is typically used to calculate the Kalman gain which could be computed based on that approximation nearly without loss of accuracy. In addition, The estimation algorithms developed are not restricted to small angles such as those restrictions found in [11], and [5]. 


\section{Development of the extended Kalman filter estimation algorithm}

The basic structure of the extended Kalman filter is

$$
\begin{aligned}
& \hat{X}_{k}^{-}=f\left(\hat{X}_{k-1}\right) \\
& P_{k}^{-}=A_{k-1} P_{k-1} A_{k-1}^{T}+Q_{k-1} \\
& K_{k}=P_{k}^{-} H_{k}^{T}\left(H_{k} P_{k}^{-} H_{k}^{T}+R_{k}\right)^{-1} \\
& \hat{X}_{k}=\hat{X}_{k}^{-}+K_{k}\left[z_{k}-\hat{z}_{k}\right] \\
& P_{k}=\left[I-K_{k} H_{k}\right] P_{k}^{-}\left[I-K_{k} H_{k}\right]^{T}+K_{k} R_{k} K_{k}^{T}
\end{aligned}
$$

where

$\hat{X}_{k}^{-} \quad$ : A priori state estimate at a time step $k$.

$A_{k} \quad:$ is the state transition matrix.

$H_{k} \quad$ : is the measurement matrix.

$\hat{X}_{k} \quad$ : A posteriori state estimate at a time step $k$.

$P_{k} \quad$ : A posteriori estimate error covariance at a time step $k$.

$Q_{k} \quad:$ is the discrete process noise covariance.

$R_{k} \quad$ : is the discrete measurement noise covariance.

$z_{k} \quad:$ is the measurement vector provided by measurement devices.

$\hat{z}_{k} \quad:$ is the estimated measurement vector.

The state transition matrix according to [9] is calculated from

$$
A_{k-1}\left(\hat{X}_{k-1}^{+}\right)=\left\{I+\left(F_{k-1}\left(\hat{X}_{k-1}^{+}\right)\right) \Delta T\right\}
$$

with $\Delta T$ defined as the sampling time interval and

$$
F_{k-1}\left(\hat{X}_{k-1}^{+}\right)=\partial f /\left.\partial X\right|_{\left(\hat{X}_{k-1}^{+}\right)}
$$

The measurement matrix $H_{k}$ is computed from

$$
H_{k}=\left.\frac{\partial h(X)}{\partial X}\right|_{X=\hat{X}}
$$

where the measurement vector $h$ corresponding to magnetometer, gyro, star sensor, and GPS is given by

$$
h=\left[\begin{array}{lllllllllllll}
b_{x b} & b_{y b} & b_{z b} & \omega_{x} & \omega_{y} & \omega_{z} & q_{1} & q_{2} & q_{3} & q_{4} & X_{I} & Y_{I} & Z_{I}
\end{array}\right]^{T}
$$

where

$b_{. b} \quad:$ is earth's magnetic field component measured by the magnetometer in the corresponding direction. The measurement matrix according to (9) and (10) are 


$$
\frac{\partial h}{\partial X}=\left[\begin{array}{ccccccccccccc}
\frac{\partial b_{x b}}{\partial X_{I}} & \frac{\partial b_{x b}}{\partial Y_{I}} & \frac{\partial b_{x b}}{\partial Z_{I}} & \frac{\partial b_{x b}}{o} & \frac{\partial b_{x b}}{o} & \frac{\partial b_{x b}}{o} & \frac{\partial b_{x b}}{\partial q_{1}} & \frac{\partial b_{x b}}{\partial q_{2}} & \frac{\partial b_{x b}}{\partial q_{3}} & \frac{\partial b_{x b}}{\partial q_{4}} & \frac{\partial b_{x b}}{\partial \omega_{x}} & \frac{\partial b_{x b}}{\partial \omega_{y}} & \frac{\partial b_{x b}}{\partial \omega_{z}} \\
\frac{\partial b_{y b}}{\partial X_{I}} & \frac{\partial b_{y b}}{\partial Y_{I}} & \frac{\partial b_{y b}}{\partial Z_{I}} & \frac{\partial b_{y b}}{o} & \frac{\partial b_{y b}}{o} & \frac{\partial b_{y b}}{o} & \frac{\partial b_{y b}}{\partial q_{1}} & \frac{\partial b_{y b}}{\partial q_{2}} & \frac{\partial b_{y b}}{\partial q_{3}} & \frac{\partial b_{y b}}{\partial q_{4}} & \frac{\partial b_{y b}}{\partial \omega_{x}} & \frac{\partial b_{y b}}{\partial \omega_{y}} & \frac{\partial b_{y b}}{\partial \omega_{z}} \\
\frac{\partial b_{z b}}{\partial X_{I}} & \frac{\partial b_{z b}}{\partial Y_{I}} & \frac{\partial b_{z b}}{\partial Z_{I}} & \frac{\partial b_{z b}}{\partial} & \frac{\partial b_{z b}}{\partial} & \frac{\partial b_{z b}}{\partial} & \frac{\partial b_{z b}}{\partial q_{1}} & \frac{\partial b_{z b}}{\partial q_{2}} & \frac{\partial b_{z b}}{\partial q_{3}} & \frac{\partial b_{z b}}{\partial q_{4}} & \frac{\partial b_{z b}}{\partial \omega_{x}} & \frac{\partial b_{z b}}{\partial \omega_{y}} & \frac{\partial b_{z b}}{\partial \omega_{z}} \\
0 & 0 & 0 & 0 & 0 & 0 & 0 & 0 & 0 & 0 & 1 & 0 & 0 \\
0 & 0 & 0 & 0 & 0 & 0 & 0 & 0 & 0 & 0 & 0 & 1 & 0 \\
0 & 0 & 0 & 0 & 0 & 0 & 0 & 0 & 0 & 0 & 0 & 0 & 1 \\
0 & 0 & 0 & 0 & 0 & 0 & 1 & 0 & 0 & 0 & 0 & 0 & 0 \\
0 & 0 & 0 & 0 & 0 & 0 & 0 & 1 & 0 & 0 & 0 & 0 & 0 \\
0 & 0 & 0 & 0 & 0 & 0 & 0 & 0 & 1 & 0 & 0 & 0 & 0 \\
0 & 0 & 0 & 0 & 0 & 0 & 0 & 0 & 0 & 1 & 0 & 0 & 0 \\
1 & 0 & 0 & 0 & 0 & 0 & 0 & 0 & 0 & 0 & 0 & 0 & 0 \\
0 & 1 & 0 & 0 & 0 & 0 & 0 & 0 & 0 & 0 & 0 & 0 & 0 \\
0 & 0 & 1 & 0 & 0 & 0 & 0 & 0 & 0 & 0 & 0 & 0 & 0
\end{array}\right]
$$

Details of computing the first three rows of equation (11) are lengthy and could be given in [1]. As evident from equation (11), there is no need to check the observability of the system because of several reasons:

1- All of the components of the attitude state vector are directly measured with a sensor in addition to magnetometer.

2- The components of the orbital state vector are the inertial position and velocity. GPS provide measurements of the satellite position, which in turn could be used to compute satellite velocity. Thus, all the components of the orbital state vector are measured through GPS, in addition to magnetometer.

\section{Errors}

Errors affecting the estimation process result from different sources. The first source is the different disturbance forces and moments affecting the spacecraft. The case study satellite is subject to many disturbances such as solar radiation pressure forces and moments, aerodynamics forces and moments, earth's oblateness till the fourth order (i.e. $J_{4}$ ), gravity gradient moments, and residual magnetic dipole moments. Modeling of these disturbance forces and moments is taken into consideration. Details of these models are given in Refs. [1], [2], [3], and [8]. The earth's magnetic field model coefficients are given in [10]. The second source of errors results from the un-modeled disturbances acting on the satellite. These disturbances are treated as a zero-mean Gaussian white process noise, $w$. This manipulation could be mathematically expressed as

$$
\stackrel{o}{X}=f(X)+B+w
$$

The third source of errors is related to the measurement process. The un-modeled measurement process is given by the measurement equation

$z=h(X)+v$

where $v$, is a white Gaussian noise associated with the measurement device. 


\section{Simulation Parameters, and Results}

The initial parameters of the case study spacecraft are $a$ (semi major axis) $=7139200 \mathrm{~m}$, $e($ orbit eccentricity $)=0, i$ (orbit inclination) $=101.085^{\circ}, \Omega$ (right ascension of ascending node) $=339.5^{\circ}, \omega$ (argument of perigee) $=69^{\circ}, v$ (true anomaly) $=6^{\circ}$, $\phi($ roll angle $)=-175^{\circ}, \psi($ yaw angle $)=180^{\circ}$, and $\theta \quad($ pitch angle $)=85^{\circ}$.

The estimated satellite parameters are initialized with $a$ (semi major axis) $=7039200 \mathrm{~m}$, $e\left(\right.$ orbit eccentricity) $=0, i$ (orbit inclination) $=98.85^{\circ}, \Omega$ (right ascension of ascending node $)=337.5^{\circ}, \omega$ (argument of perigee) $=69^{\circ}$, and $v$ (true anomaly) $=0^{\circ}$, $\phi($ roll angle $)=0^{\circ}, \psi($ yaw angle $)=0^{\circ}$, and $\theta$ (pitch angle $)=0^{\circ}$.

Epoch time (1/4/2013 0h:0m:0s). Time step $(\Delta T)=4$ seconds.

The continuous measurement noise covariance matrix is given by

$R=\left[\begin{array}{cccc}R_{11} & 0_{3 * 3} & 0_{3 * 4} & 0_{3 * 3} \\ 0_{3 * 3} & R_{22} & 0_{3 * 4} & 0_{3 * 3} \\ 0_{4 * 3} & 0_{4 * 3} & R_{33} & 0_{4 * 3} \\ 0_{3 * 3} & 0_{3 * 3} & 0_{3 * 4} & R_{44}\end{array}\right]$

with

$R_{11}=\left(\frac{50}{3} \times 10^{-9}\right)^{2} I_{3 \times 3}, \quad R_{22}=\left(\frac{0.02}{3} \times \frac{\pi}{180 \times 60 \times 60}\right)^{2} I_{3 \times 3}, \quad R_{33}=\left(\frac{0.000484814}{3}\right)^{2} I_{4 \times 4}, \quad$ and $R_{44}=\left(\frac{50}{3}\right)^{2} \times I_{3 \times 3}$

The continuous process noise covariance matrix is given by

$$
Q=\left[\begin{array}{cc}
Q_{11} & 0_{6 \times 7} \\
0_{7 \times 6} & Q_{22}
\end{array}\right]
$$

where

$Q_{11}=\left[\begin{array}{cc}Q_{33} & 0_{3 \times 3} \\ 0_{3 \times 3} & Q_{44}\end{array}\right], \quad Q_{33}=\left(30 \times 10^{3}\right)^{2} \times I_{3 \times 3}, \quad$ and $\quad Q_{44}=26^{2} I_{3 \times 3}, \quad Q_{22}=\left[\begin{array}{cc}Q_{55} & 0_{4 \times 3} \\ 0_{3 \times 4} & Q_{66}\end{array}\right]$, $Q_{55}=\left(0.001 \times \frac{\pi}{180}\right)^{2} \times I_{4 \times 4}, Q_{66}=\left(5 \times 10^{-5}\right)^{2} \times I_{4 \times 4}$,

The initial estimation error covariance matrix is given by

$$
P_{0}=\left[\begin{array}{cc}
P_{11} & 0_{6 \times 7} \\
0_{7 \times 6} & P_{22}
\end{array}\right]
$$

where

$$
\begin{aligned}
& P_{11}=\left[\begin{array}{cc}
P_{33} & 0_{3 \times 3} \\
0_{3 \times 3} & P_{44}
\end{array}\right], \quad P_{33}=\left(30 \times 10^{3}\right)^{2} \times I_{3 \times 3}, \text { and } P_{44}=26^{2} I_{3 \times 3}, \quad P_{22}=\left[\begin{array}{cc}
P_{55} & 0_{4 \times 3} \\
0_{3 \times 4} & P_{66}
\end{array}\right], \quad P_{55}=\left(\frac{1}{3}\right)^{2} \times I_{4 \times 4}, \\
& P_{66}=\left(\frac{2}{3} \times \frac{1}{\Delta T}\right)^{2} \times I_{4 \times 4},
\end{aligned}
$$


The estimation error of attitude angles is shown in Fig. 1. As clear in this figure, the estimation error is converging to near zero within about 10 seconds despite large initial attitude estimation error. Fig. 2 shows the angular velocity estimation error. Figure 3 shows the magnitude of the position estimation error between the true and estimated satellites. As shown in this figure the estimation error is reduced drastically before 5 seconds. The maximum standard deviation of the attitude angles estimation error was $0.11^{\circ}$. The standard deviation of the magnitude of the position estimation error is about $9.9 \mathrm{~m}$.
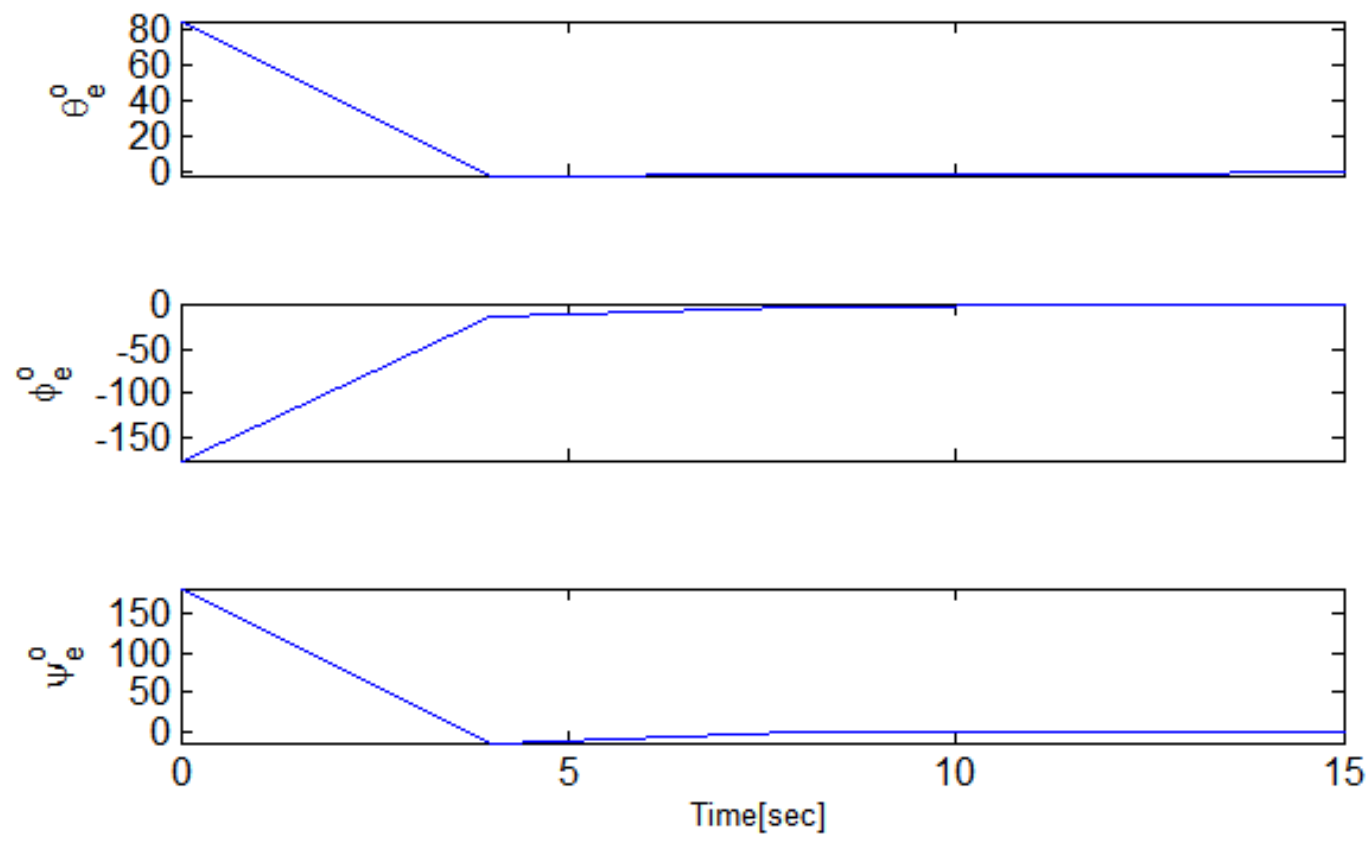

Fig. 1. Attitude estimation error.
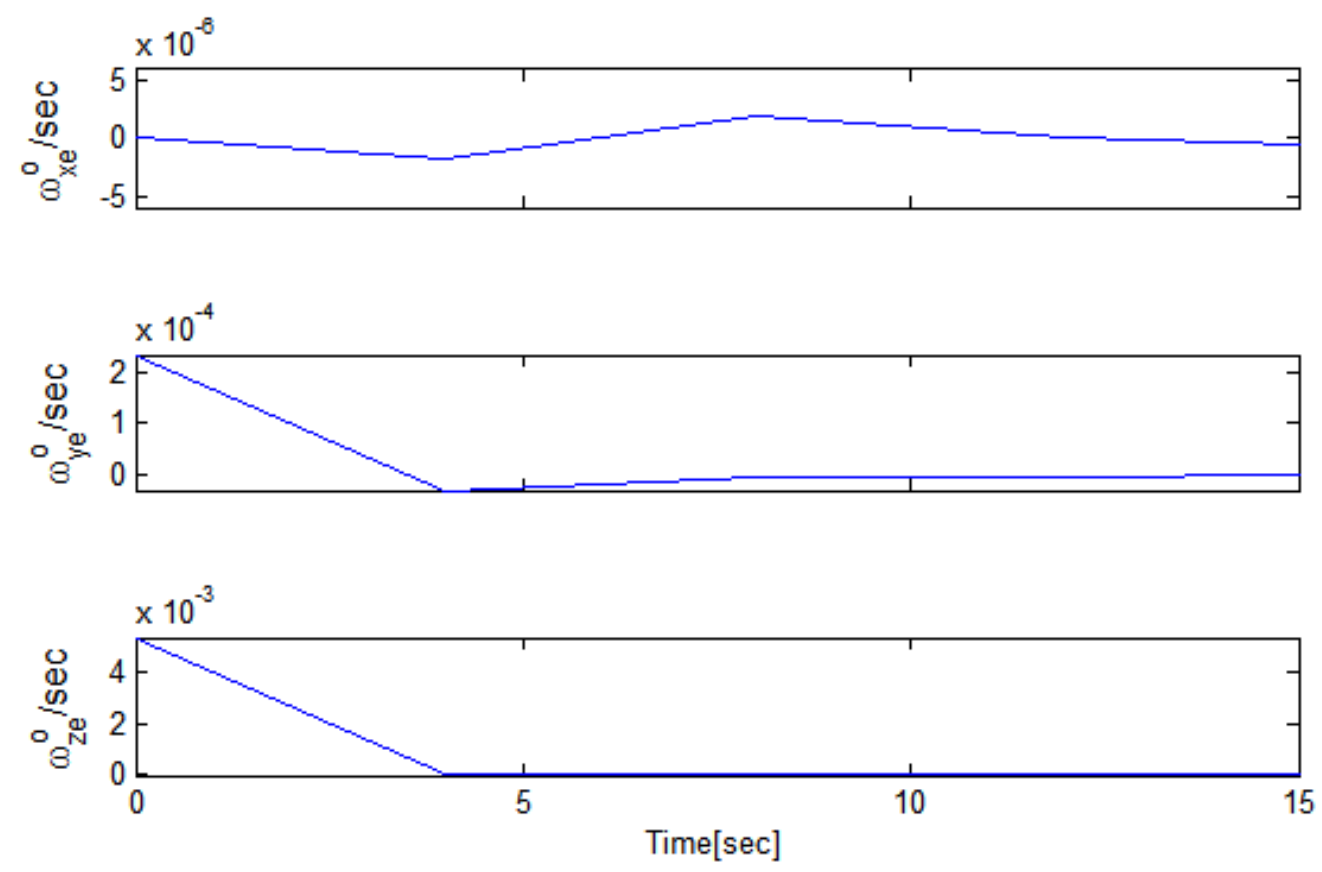

Fig. 2. Angular velocity estimation error. 


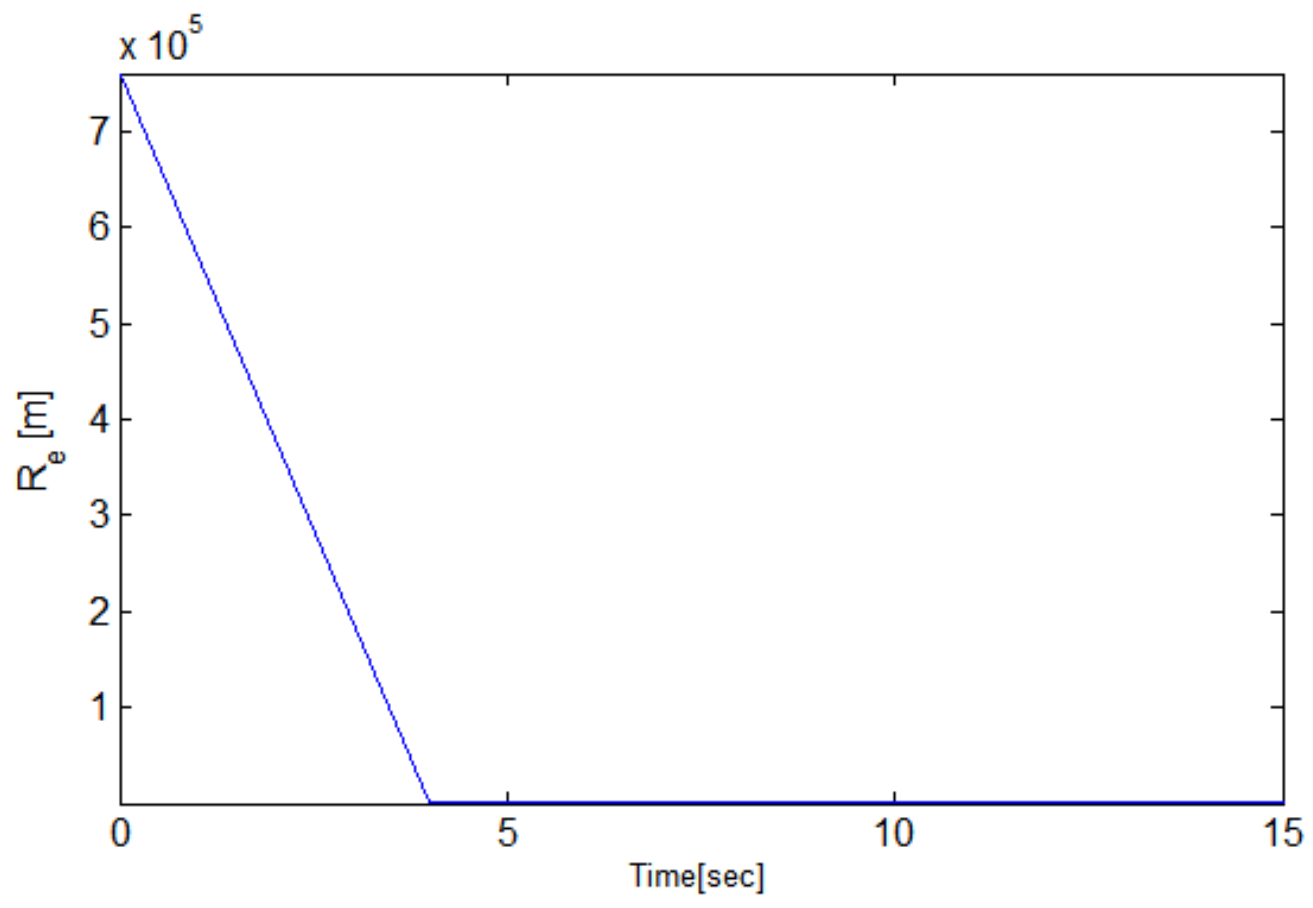

Fig. 3. Magnitude of position estimation error.

\section{Conclusion}

The proposed structure of the estimation algorithm had successfully extended the capability of the work done in [1] as seen in Table 1.

The developed estimation algorithm converged within less than ten seconds despite large initial estimation error. The estimation algorithm developed is characterized by fast convergence (typically within ten seconds), high accuracy $\left(0.11^{\circ}\right.$ for estimation errors and $10 \mathrm{~m}$ for position error), and the capability to deal with large initial estimation errors (as high as $180^{\circ}$ ). Therefore, it is considered to be suitable during imaging and before execution of any orbital maneuver.

Table 1. Enhancements of the algorithm of Ref. [1].

\begin{tabular}{l|c|c}
\hline \hline & Algorithm developed in [1] & Current research algorithm \\
\hline Convergence time & $8000 \mathrm{sec}$ & $10 \mathrm{sec}$ \\
\hline Attitude estimation accuracy & 5 degrees & 0.11 degrees \\
\hline Orbit estimation accuracy & $115 \mathrm{~km}$ & $9.9 \mathrm{~m}$ \\
\hline Sensors & Magnetometer & $\begin{array}{c}\text { Magnetometer, GPS, Gyro, } \\
\text { Star Sensor, Gyro }\end{array}$ \\
\hline $\begin{array}{l}\text { Capability to deal with large } \\
\text { initial estimation errors }\end{array}$ & Capable & $\begin{array}{c}\text { Capable } \\
\text { Satellite operation mode }\end{array}$ \\
\hline \hline
\end{tabular}




\section{References}

[1] Tamer, M., "New Algorithms of Nonlinear Spacecraft Attitude Control via Attitude, Angular Velocity, and Orbit Estimation Based on the Earth's Magnetic Field", PhD Thesis, Cairo University, 2009.

[2] Mekky, T., "The Global Positioning System Application to Satellite Position and Attitude Determination", MSc thesis, Aerospace Department, Cairo University, 2003.

[3] Wertz, J. R., "Spacecraft Attitude Determination and Control", D. Reidel Publishing Company, 1997.

[4] Shorshi, G., Itzhack Y. Bar-Itzhack, "Satellite Autonomous Navigation Based on Magnetic Field Measurements", Journal of Guidance Control and Dynamics, Vol.18, No.4, 1995, pp. 843-850.

[5] Deutschmann, J., Itzhack Y. Bar-Itzhack, "Evaluation of Attitude and Orbit Estimation Using Actual Earth Magnetic Field Data", Journal of Guidance Control and Dynamics, Vol.24, No.3, 2001, pp. 616-623.

[6] Ersin, H., Hajiyev C., "Pico satellite attitude estimation via Robust Unscented Kalman Filter in the presence of measurement faults", ISA Transactions, Vol.49, 2010, pp. 249256.

[7] Jizheng, C., Jianping Y., Qun , F.,"Flight Vehicle Attitude Determination Using the Modified Rodrigues Parameters", Chinese Journal of Aeronautics, Vol.21, 2008, pp. 433-440.

[8] Larson, W., and Wertz, J. R., "Space Mission Analysis and Design”, Microcosm Press, and Kluwer Academic Publisher, 1999.

[9] Shorshi, G., Itzhack Y. Bar-Itzhack, "Satellite Autonomous Navigation Based on Magnetic Field Measurements", Journal of Guidance Control and Dynamics, Vol.18, No.4, 1995, pp. 843-850.

[10] Maus, S., et al, “The US/UK World Magnetic Model for 2010-2015”, NOAA Technical Report, NESDIS/NGDC-1.

[11] Si Mohammed, A., et al "Simulation of microsatellite attitude using Kalman filtering", Simulation Modeling practice and theory, Vol.16, 2008, pp. 257-277. 